TRANSACTIONS OF THE

AMERICAN MATHEMATICAL SOCIETY

Volume 353, Number 10, Pages 4139-4154

S 0002-9947(01)02761-1

Article electronically published on June 1, 2001

\title{
HYPERBOLIC CONSERVATION LAWS WITH STIFF REACTION TERMS OF MONOSTABLE TYPE
}

\author{
HAITAO FAN
}

ABSTRACT. In this paper the zero reaction limit of the hyperbolic conservation law with stiff source term of monostable type

$$
\partial_{t} u+\partial_{x} f(u)=\frac{1}{\epsilon} u(1-u)
$$

is studied. Solutions of Cauchy problems of the above equation with initial value $0 \leq u_{0}(x) \leq 1$ are proved to converge, as $\epsilon \rightarrow 0$, to piecewise constant functions. The constants are separated by either shocks determined by the Rankine-Hugoniot jump condition, or a non-shock jump discontinuity that moves with speed $f^{\prime}(0)$. The analytic tool used is the method of generalized characteristics. Sufficient conditions for the existence and non-existence of traveling waves of the above system with viscosity regularization are given. The reason for the failure to capture the correct shock speed by first order shock capturing schemes when underresolving $\epsilon>0$ is found to originate from the behavior of traveling waves of the above system with viscosity regularization.

\section{INTRODUCTION}

In this paper we study hyperbolic equations with stiff source term of monostable type

$$
\begin{aligned}
& \partial_{t} u+\partial_{x} f(u)=\frac{1}{\epsilon} u(1-u), \\
& u(x, 0)=u_{0}(x),
\end{aligned}
$$

where $\epsilon>0$ is the reaction time. We call the source term of (1.1) monostable in the sense that one of the two equilibria of (1.1), $u=1$, is stable while the other, $u=0$, is unstable. Since most equations governing reacting flows or dynamics of phase transitions are combinations of inhomogeneous fluid dynamics equations and reaction-diffusion equations [ $\mathrm{AK}, \mathrm{VK}]$, equation (1.1) can serve as a prototype model to study issues involved in reacting flows. In a typical reacting flow, the reacting time $\epsilon$ is very small. This motivates us to investigate the behavior in the $\epsilon \rightarrow 0+$ limit of (1.1) in this paper.

We shall also consider the viscosity regularization of (1.1),

$$
\partial_{t} u+\partial_{x} f(u)=\frac{1}{\epsilon} u(1-u)+A \epsilon u_{x x},
$$

Received by the editors October 28, 1999 and, in revised form, June 19, 2000.

2000 Mathematics Subject Classification. Primary 35L65, 35B40, 35B25.

Key words and phrases. Conservation law with source term, reaction-convection-diffusion equation, zero reaction time limit.

Research supported in part by NSF grant No. DMS 9705732. 
and its traveling waves, where $A>0$ is a constant. The viscosity term arises naturally in reactive flows. Numerical schemes for (1.1) actually mimic (1.2) due to numerical viscosity. We shall see that the behavior of the traveling waves of (1.2) yields the explanation of the difficulties we encountered in the numerical computations of (1.1) when we try to underresolve $\epsilon>0$.

In this paper, we assume the following:

$\left(H_{1}\right) \cdot f^{\prime \prime}(u)>0$.

$\left(H_{2}\right)$. Initial datum $u_{0}(x)$ satisfies $u_{0}(x) \in C(\mathbb{R} ; \mathbb{R}), 0 \leq u_{0}(x) \leq 1$ and the set $\left\{x \in \mathbb{R} \mid u_{0}(x)=0\right\}$ is the union of finitely many disjoint closed intervals:

$$
\left\{x \in \mathbb{R} \mid u_{0}(x)=0\right\}=\bigcup_{j=1}^{N}\left[a_{j}, b_{j}\right] .
$$

From the classical theory of conservation laws $[\mathrm{Kru}$, we know that there is a unique admissible solution of (1.1) in BV space for each fixed $\epsilon$. When $f^{\prime \prime}>0$, a solution $u(x, t)$ of $(1.1)$ in $\mathrm{BV}$ is called admissible if $u(x-, t) \geq u(x+, t)$ holds for all $(x, t)$ in the domain of the definition of $u$. The requirement $\left(H_{2}\right)$ is mostly for simplicity of presentation. With these assumptions, we establish the following results:

Theorem 1.1. Let $u^{\epsilon}$ be the solution of (1.1). Under the assumptions $\left(H_{1}\right)$ and $\left(H_{2}\right)$, the limit

$$
u(x, t)=\lim _{\epsilon \rightarrow 0+} u^{\epsilon}(x, t)
$$

exists for almost all $(x, t) \in \mathbb{R} \times \mathbb{R}^{+}$. The value of $u(x \pm, t)$ is either 1 or 0 . Furthermore, there are $n$ curves $a_{j}(t)$ and $b_{j}(t)$ defined on $\left[0, T_{j}\right], j=1,2, \ldots, n$, such that

(i) the set $\{x \in \mathbb{R} \mid u(x, t)=0\}=\bigcup_{j=1}^{n}\left(a_{j}(t), b_{j}(t)\right)$ and intervals $\left(a_{j}(t), b_{j}(t)\right)$, $j=1,2, \ldots, n$, are pairwise disjoint;

(ii) $b_{j}(t)=b_{j}+f^{\prime}(0) t$

(iii) the curve $a_{j}(t)$ satisfies the Rankine-Hugoniot condition and hence

$$
a_{j}(t)=a_{j}+(f(1)-f(0)) t
$$

(iv) at the end point $t=T_{j}$ of the domain of the definition of $a_{j}(t)$ and $b_{j}(t)$, $a_{j}\left(T_{j}\right)=b_{j}\left(T_{j}\right)$.

Theorem 1.1 reveals that, as $\epsilon \rightarrow 0$, there are two types of discontinuities that will connect the constant equilibrium states 1 and 0 . The first type is classical shock wave, as described in (iii), that propagate with speed determined by the RankineHugoniot jump condition and satisfy the entropy condition for the homogeneous equation $\partial_{t} u+\partial_{x} f(u)=0$. The other type of discontinuity, called non-shock jump discontinuity throughout this paper, is described by (ii) in Theorem 1.1. This discontinuity violates the Rankine-Hugoniot jump condition and the entropy condition for the homogeneous equation.

We also obtained results on the traveling waves of (1.2):

Theorem 1.2. If $f^{\prime \prime}>0$ and

$$
c>f^{\prime}(1)+\sqrt{4 A+\left(f^{\prime}(1)-f^{\prime}(0)\right)^{2} / 4},
$$

then there is a traveling wave of (4.1) with speed $c>0$. Furthermore, if 


$$
c<f^{\prime}(0)+2 \sqrt{A}
$$

then there is no traveling wave of (1.2) satisfying $0 \leq u \leq 1$.

Remark. The theorem above states that there are traveling waves of (1.2) if $c>0$ is sufficiently larger than $f^{\prime}(1)$. We note that the shock wave

$$
u(x, t)= \begin{cases}0, & \text { if } x>(f(1)-f(0)) t, \\ 1, & \text { if } x<(f(1)-f(0)) t\end{cases}
$$

is a traveling wave of (1.1) slower than $f^{\prime}(1)$. Then it is natural to ask whether there are traveling waves of (1.2) with speed smaller than $f^{\prime}(1)$ and if there is such a traveling wave, whether it approaches (1.5) as $A \rightarrow 0$. These questions will be left for future investigation.

Remark. Equation (1.2) is closely related to the KPP or Fisher equation

$$
u_{t}=u_{x x}+u(1-u) \text {. }
$$

Results and methods for (1.6) will certainly shed light on the research of (1.2). It is well known that (1.6) has traveling waves if and only if the speed of the wave $c \geq 2$. Solutions of initial value problems of (1.6) converge in form to traveling waves. If the initial data of (1.6) decays faster as $x \rightarrow \infty$, then the solution tends to converge to a slower traveling wave. For precise statements on the convergence to traveling waves of the solution of (1.6), see $[\mathrm{Br}]$.

Remark. It is possible that as $A \rightarrow 0+$, the solution of (1.2) for fixed initial data approaches that of (1.1) with the same initial data. It is then worth noting that although (1.2) has traveling waves for $c>0$ large enough, equation (1.1) does not have so many traveling waves. This indicates that some traveling waves of (1.2) do not have to approach those of (1.1), if they exist. This is different from the behavior of conservation laws without monostable source terms.

From the original modeling of (1.1), there are some requirements that numerical schemes for (1.1) should satisfy: In the original modeling for (1.1), $u$ typically represents mass fractions of reactant and hence it is not physically meaningful outside the range $0 \leq u \leq 1$. In other words, numerical schemes of (1.1) should preserve the invariant region of (1.1), $0 \leq u \leq 1$. Furthermore, consider the case of liquid/vapor transitions (see $\mathrm{Fan}$ ), where $u$ is the mass fraction of liquid in liquid/vapor mixture. We see that a numerical scheme should also satisfy the metastability condition: If $u_{0} \equiv 0$, then $u(x, t) \equiv 0$. Failure to satisfy the metastability requirement will result in substantial error in the wave speeds of (1.1) and (1.2). The following first order partially implicit method satisfies these two requirements:

$$
u_{j}^{n+1}=u_{j}^{n}-\frac{\Delta t}{\Delta x}\left(f\left(u_{j}\right)-f\left(u_{j-1}\right)\right)+\frac{\Delta t}{\epsilon} u^{n}\left(1-u^{n+1}\right) .
$$

Since (1.1) is stiff, a practical numerical method for such a problem would require an underresolved temporal discretization (time step $\Delta t$ much bigger than the reaction time $\epsilon$ ). However, doing so in shock capturing methods leads to incorrect shock speed $[\overline{\mathrm{BKT}}],[\mathrm{CMR},[\mathrm{LY}]$. We conducted numerical tests using (1.7) and initial data

$$
u(x, 0)= \begin{cases}0, & \text { if } x>0 \\ 1, & \text { if } x<0\end{cases}
$$


The exact solution should be a shock of speed $c=f(1)-f(0)$ :

$$
u(x, t)= \begin{cases}0, & \text { if } x-c t>0 \\ 1, & \text { if } x-c t<0\end{cases}
$$

Along with parameters and functions used in the numerical test are $\epsilon=0.0005$, $\Delta x=0.002, \Delta t=0.0005$ and $f(u)=u^{2} / 2+u$. The speed of the shock in the numerical solution is about 2.47 , substantially different from the correct speed $c=f(1)-f(0)=1.5$. In fact, Theorem 1.2 already predicts this discrepancy: With a first order scheme, numerical viscosity is proportional to $\Delta x+\Delta t$. This corresponds to the case when $A=O(1)(\Delta t+\Delta t) / \epsilon$ in (1.2). If we underresolve $\epsilon>0$, then the corresponding constant $A$ in (1.2) is not small. If $f^{\prime}(1)<2 \sqrt{A}$, then by Theorem 1.2, the traveling wave speed must be bigger than the correct shock speed $f(1)-f(0)$. Thus, schemes with first order numerical viscosity cannot give us the correct shock speed of (1.1) if $\epsilon>0$ is underresolved. On the other hand, if the numerical viscosity can be made much smaller than $\epsilon$, then the stability behavior of KPP suggests that it is possible to obtain the correct speed. This points out the need for higher order schemes for (1.1) that still satisfy the invariant region and metastability requirements. Whether there are such higher order methods and whether such higher order schemes can produce the correct shock speed when underresolving $\epsilon>0$ are left for future research. Bao and Jin [BJ], developed a random projection method that gives the correct shock speeds for

$$
\partial_{t} u+\partial_{x} f(u)=\frac{1}{\epsilon} g(u),
$$

for $g(u)=u\left(1-u^{2}\right)$ and $u(1-u)$, even when $\epsilon>0$ is underresolved.

A brief review of related results is in order: Many authors, [FH1, [FH2, [Har, Lyb], Mas, MS], Sin1, Sin2], [Sin3, have studied the large time behavior and attractors of (1.10) under certain assumptions on $g(u)$ and focused on periodic initial data. The issues and limiting processes as well as the behavior of (1.1) investigated in this paper are different from those studied in the references cited above. For example, the non-shock discontinuity provided by (iii) of Theorem 1.1 is not present in those studies. The behavior of traveling waves of (1.2) is also different from what we already know from the references cited above. Recently, Fan, Jin and Teng [FJT] studied $\epsilon \rightarrow 0+$ limiting behavior of (1.10) with bistable source term $g(u)=u\left(1-u^{2}\right)$ with initial data having only finitely many zeros, where 0 is the unstable equilibrium of the source term, and proved the convergence of solutions of (1.10) when $\epsilon \rightarrow 0$. In this paper, the source term is of monostable type, $g(u)=u(1-u)$ and the initial data $u_{0}(x)$ is equal to an unstable equilibrium in several intervals. The behavior of the bistable traveling wave of (1.10) with viscosity is quite different from that of (1.2).

This paper is organized as follows: In Section 2, we recall some basic properties of generalized characteristics for conservation laws. We shall use them in our study of (1.1) in the $\epsilon \rightarrow 0+$ limit. In Section 3, we shall prove Theorem 1.1. In Section 4 we shall establish Theorem 1.2. 


\section{Preliminaries}

A Lipschitzian curve $x=\xi(t)$ defined on an interval $[a, b]$ is called a characteristic curve associated to the solution $u(x, t)$ of (1.1) if, for almost all $t \in[a, b]$,

$$
\frac{d \xi}{d t} \in\left[f^{\prime}(u(\xi(t)+, t)), f^{\prime}(u(\xi(t)-, t))\right] .
$$

From Fil], for any $(\bar{x}, \bar{t}) \in \mathbb{R} \times(0, \infty)$, there exists at least one backward characteristic $\xi(t ; \bar{x}, \bar{t})$ defined on a maximal interval $(s, \bar{t}], s \geq 0$, with $\xi(\bar{t} ; \bar{x}, \bar{t})=\bar{x}$. The set of all backward characteristics through $(\bar{x}, \bar{t})$ form a funnel confined between the minimal and the maximal backward characteristics through $(\bar{x}, \bar{t})$. We denote the minimal and maximal backward characteristics by $\xi_{-}(t ; \bar{x}, \bar{t})$ and $\xi_{+}(t ; \bar{x}, \bar{t})$ respectively. The following Lemmas 2.1, 2.2 and 2.3 are from [Daf]:

Lemma 2.1. The extremal backward characteristic $\xi_{ \pm}(t ; \bar{x}, \bar{t})$ associated with the solution $u(x, t)$ of (1.1) satisfies, for $t \in(s, \bar{t}]$,

$$
\begin{aligned}
& \frac{d \xi}{d t}=f^{\prime}(v(t)), \\
& \frac{d v}{d t}=\frac{1}{\epsilon} v\left(1-v^{2}\right),
\end{aligned}
$$

with initial conditions

$$
\left(\xi_{-}(\bar{t} ; \bar{x}, \bar{t}), v(\bar{t})\right)=(\bar{x}, u(\bar{x}-, \bar{t}))
$$

and

$$
\left(\xi_{+}(\bar{t} ; \bar{x}, \bar{t}), v(\bar{t})\right)=(\bar{x}, u(\bar{x}+, \bar{t}))
$$

respectively. Furthermore, for both $\xi(t):=\xi_{-}(t)$ and $\xi(t):=\xi_{+}(t)$, equations

$$
v(t)=u(\xi(t)-, t)=u(\xi(t)+, t)
$$

hold for almost all $t \in(s, \bar{t}]$.

Lemma 2.2. Any two extremal backward characteristics do not intersect.

Lemma 2.3. If the solution $|u(x, t)| \leq C$ for some constant $C>0$, then backward characteristics associated with $u(x, t)$ are defined on $[0, \bar{t}]$.

Lemma 2.4. If $f \in C^{1}(\mathbb{R} ; \mathbb{R})$ and $1 \geq u_{0}(x) \geq 0$, then the solution of (1.1) satisfies

$$
1 \geq u(x, t) \geq 0 \text { for } t>0 .
$$

Proof. According to $\mathrm{Kru}$, for any fixed $\epsilon$, the solution $u(x, t)$ is the $\mu \rightarrow 0+$ limit of solutions $u(x, t ; \mu)$ of

$$
\begin{aligned}
& \partial_{t} u+\partial_{x} f(u)=\frac{1}{\epsilon} u(1-u)+\mu \partial_{x}^{2} u, \\
& u(x, 0, \mu)=u_{0}(x) .
\end{aligned}
$$

It is easy to see that the maximum and minimum principle hold in the region $u>1$ and $1>u \geq 0$ respectively. Thus the solution $u(x, t ; \mu)$ satisfies $(2.5)$. Therefore the limit $u(x, t)=\lim _{\mu \rightarrow 0+} u(x, t ; \mu)$ also satisfies $(2.5)$.

The following corollary immediately follows Lemma 2.3 and 2.4:

Corollary 2.5. Backward characteristics through the point $(\bar{x}, \bar{t})$ are defined on $[0, \bar{t}]$. 
We say that a curve $x=\zeta(t)$ crosses the curve $x=\xi(t)$ from the left (right) as $t$ decreases if there is a $t_{0}$ in the domains of definition of $\zeta$ and $\xi$ such that $\zeta\left(t_{0}\right)=\xi\left(t_{0}\right)$ and $\zeta\left(t_{0}+\delta\right)<(>) \xi\left(t_{0}+\delta\right), \zeta\left(t_{0}-\delta\right)>(<) \xi\left(t_{0}-\delta\right)$ for sufficiently small $\delta>0$.

Lemma 2.6. From any point $(\bar{x}, \bar{t}), \bar{t}>0$, there is a unique forward generalized characteristic $\zeta(t ; \bar{x}, \bar{t})$ of $(1.1)$ defined as

$$
\begin{aligned}
& \frac{d \zeta}{d t}= \begin{cases}f^{\prime}(u(\zeta(t), t)), & \text { if } u(\zeta(t)-, t)=u(\zeta(t)+, t), \\
\frac{f(u(\zeta(t)-, t))-f(u(\zeta(t)+, t))}{u(\zeta(t)-, t)-u(\zeta(t)+, t)}, & \text { if } u(\zeta(t)-, t)>u(\zeta(t)+, t),\end{cases} \\
& \zeta(\bar{t})=\bar{x}, t>\bar{t} .
\end{aligned}
$$

Furthermore, a minimal backward characteristics $x=\xi_{-}(t)$ of (1.1) cannot cross $x=\zeta(t)$ from the left as $t$ decreases. Similarly, maximal backward characteristics $x=\xi_{+}(t)$ of (1.1) cannot cross $x=\zeta(t)$ from the right as $t$ decreases.

Proof. The existence and uniqueness of the forward generalized characteristics $\zeta(t ; \bar{x}, \bar{t})$ is stated and proved in Theorem 3.4 of [Daf]. To see that $\xi_{-}(t)$ cannot $\operatorname{cross} \zeta(t)$ from the left as $t$ decreases, we note that if $\zeta(t)=\xi_{-}(t)$, it would hold that

$$
\begin{aligned}
\frac{d \zeta}{d t} & = \begin{cases}f^{\prime}(u(\zeta(t), t)), & \text { if } u(\zeta(t)-, t)=u(\zeta(t)+, t), \\
\frac{f(u(\zeta(t)-, t))-f(u(\zeta(t)+, t))}{u(\zeta(t)-, t)-u(\zeta(t)+, t)}, & \text { if } u(\zeta(t)-, t)>u(\zeta(t)+, t),\end{cases} \\
& \leq f^{\prime}(u(\zeta(t)-, t))=\frac{d \xi_{-}}{d t} .
\end{aligned}
$$

The proof for the corresponding statement for $\xi_{+}(t)$ is similar.

\section{Proof of Theorem 1.1}

In this section, we shall prove that admissible solution of (1.1) converges as $\epsilon \rightarrow 0+$. By performing the transformation $x \mapsto x-f^{\prime}(0) t$ in (1.1) if necessary, we can assume $f^{\prime}(0)=0$ without loss of generality.

First, we investigate the structure of the solution $u^{\epsilon}(x, t)$ of (1.1).

Lemma 3.1. Let $u(x, t)$ be the solution of (1.1) with initial value $u_{0}(x)$ satisfying assumption $\left(H_{2}\right)$. Then there are curves $a_{1}^{\epsilon}(t)<b_{1}^{\epsilon}(t)<a_{2}^{\epsilon}(t)<b_{2}^{\epsilon}(t)<\ldots<b_{n}^{\epsilon}(t)$, with $a_{j}^{\epsilon}(t)$ and $b_{j}^{\epsilon}(t)$ defined on $\left[0, T_{j}\right]$, such that

$$
\left\{x \in \mathbb{R} \mid u^{\epsilon}(x+, t)=0 \text { or } u^{\epsilon}(x-, t)=0\right\}=\bigcup_{j=1}^{n}\left[a_{j}^{\epsilon}(t), b_{j}^{\epsilon}(t)\right] .
$$

Moreover, $a_{j}^{\epsilon}(0)=a_{j}$ and $b_{j}^{\epsilon}(t) \equiv b_{j}$ and $\left(a_{j}^{\epsilon}(\bar{t}), b_{j}^{\epsilon}(\bar{t})\right) \subseteq\left(a_{j}, b_{j}\right)$. Furthermore, the curves $a_{j}^{\epsilon}(t)$ and $b_{j}^{\epsilon}(t), j=1,2, \ldots, n$, are Lipschitz continuous with Lipschitz constant $\leq f^{\prime}(1)$.

Proof. For each fixed $j \in\{1,2, \ldots, n\}$, pick a point $\bar{x} \in\left(a_{j}, b_{j}\right)$. The minimal backward characteristic issued from $(\bar{x}, \bar{t}), \xi^{\epsilon}(t, \bar{x}, \bar{t})$, is determined by

$$
\begin{aligned}
& \frac{d \xi}{d t}=f^{\prime}(v(t)), \\
& \frac{d v}{d t}=\frac{1}{\epsilon} v(1-v), \\
& (\xi(\bar{t} ; \bar{x}, \bar{t}), v(\bar{t}))=(\bar{x}, u(\bar{x}-, \bar{t})) .
\end{aligned}
$$


In (3.2) and the rest of proof of this lemma, we drop $\epsilon$ when no confusion is expected. From (3.2) and the assumption in $\left(H_{2}\right), 0 \leq u_{0}(x) \leq 1$, we see that $0 \leq v(t) \leq 1$. Then $(3.2)_{1}$ yields $|\bar{x}-\xi(0 ; \bar{x}, \bar{t})| \leq f^{\prime}(1) \bar{t}$ which implies that $\xi(0 ; \bar{x}, \bar{t}) \in\left(a_{j}, b_{j}\right)$ for small enough $\bar{t}>0$, and hence $u(\bar{x}-, \bar{t})=0$. Since two extremal backward characteristics do not intersect, the above reasoning implies further that for small $\bar{t}>0$, there is an interval $(a, b)$ containing $\bar{x}$ such that $u(x, \bar{t})=0$ for $x \in(a, b)$. Let $\left(a_{j}^{\epsilon}(\bar{t}), b_{j}^{\epsilon}(\bar{t})\right)$ denotes the maximum of such intervals. For any $x \in\left(a_{j}^{\epsilon}(\bar{t}), b_{j}^{\epsilon}(\bar{t})\right)$, we have $\xi(t ; x, \bar{t}) \equiv x$ and $u(x, t)=u_{0}(x)=0$ for $0<t<\bar{t}$. This implies that

$$
\left(a_{j}^{\epsilon}(\bar{t}), b_{j}^{\epsilon}(\bar{t})\right) \subseteq\left(a_{j}, b_{j}\right)
$$

for if otherwise, there would be some point $x \in\left(a_{j}^{\epsilon}(\bar{t}), b_{j}^{\epsilon}(\bar{t})\right)$ such that $u_{0}(x) \neq 0$, which contradicts what we just proved. It is clear from the definition of $a_{j}^{\epsilon}(t)$ and $b_{j}^{\epsilon}(t)$ that $a_{j}^{\epsilon}(0)=a_{j}$ and $b_{j}^{\epsilon}(0)=b_{j}$. To prove (3.1), it remains to prove that $u(\bar{x} \pm, \bar{t}) \neq 0$ for $\bar{x} \notin \bigcup_{j=1}^{n}\left[a_{j}^{\epsilon}(\bar{t}), b_{j}^{\epsilon}(\bar{t})\right]$. To this end, we see that if $u(\bar{x}+, \bar{t})=0$ (or $u(\bar{x}-, \bar{t})=0$ ), then the maximal characteristics (or minimal characteristics) issued from $(\bar{x}, \bar{t}), \xi(t ; \bar{x}, \bar{t}) \equiv \bar{x}$ and $u_{0}(\bar{x})=0$ Thus, $\bar{x} \in\left[a_{j}, b_{j}\right]$ for some $j \in\{1,2, \ldots, n\}$. If $\bar{x}<a_{j}^{\epsilon}(\bar{t})$, then we can pick any point $x_{1} \in\left(a_{j}^{\epsilon}(\bar{t}), b_{j}^{\epsilon}(\bar{t})\right)$. For any point $x \in$ $\left(\bar{x}, x_{1}\right)$, according to Lemma 2.2 , we have $a_{j} \leq \xi(0 ; \bar{x}, \bar{t}) \leq \xi(0 ; x, \bar{t}) \leq \xi\left(0 ; x_{1}, \bar{t}\right) \leq b_{j}$ and hence $u(x, \bar{t})=0$ for all $x \in\left[\bar{x}, x_{1}\right]$. This violates the definition of $a_{j}^{\epsilon}(\bar{t})$ and therefore $\bar{x} \geq a_{j}^{\epsilon}(\bar{t})$. Similarly, we can prove that $\bar{x} \leq b_{j}^{\epsilon}(\bar{t})$. This proves $(3.1)$.

We already proved $b_{j}(t) \leq b_{j}$ for $T_{j} \geq t>0$. To prove $b_{j}(t) \equiv b_{j}$, it suffices to prove that $b_{j}(t)<b_{j}$ is impossible. To this end, we assume the contrary, i.e. $b_{j}(\bar{t})<b_{j}$ for some $\bar{t}>0$. By definition of $b_{j}(t)$, there is a constant $\delta>0$ such that $u(\bar{x}, \bar{t})>0$ for $\bar{x} \in\left(b_{j}(\bar{t}), b_{j}(\bar{t})+\delta\right)$ with $b_{j}(\bar{t})+\delta<b_{j}$. Then, from (3.2), we have

$$
b_{j} \leq \xi(0 ; \bar{x}, \bar{t}) \leq \bar{x}<b_{j}(\bar{t})+\delta<b_{j}
$$

which is a contradiction. This proves $b_{j}(t) \equiv b_{j}$.

To prove that the functions $a_{j}^{\epsilon}(t), j=1,2, \ldots, n$, are Lipschitz continuous, we consider

$$
\frac{a_{j}^{\epsilon}(\bar{t})-a_{j}^{\epsilon}(t)}{\bar{t}-t}
$$

for arbitrarily chosen $T_{j} \geq \bar{t}>t \geq 0$. It is clear that $u\left(a_{j}^{\epsilon}(\bar{t})+, \bar{t}\right)=0$ and hence $u\left(\xi_{+}\left(t ; a_{j}^{\epsilon}(\bar{t}), \bar{t}\right), t\right)=0$ for all $t<\bar{t}$ from (3.1). Thus, we have

$$
a_{j}^{\epsilon}(t) \leq \xi_{+}\left(t ; a_{j}^{\epsilon}(\bar{t}), \bar{t}\right) \equiv a_{j}^{\epsilon}(\bar{t}) .
$$

Choose $x_{1}$ sufficiently close to $a_{j}^{\epsilon}(\bar{t})$ such that $u\left(x_{1}, \bar{t}\right)>0$ and $x_{1}<a_{j}^{\epsilon}(\bar{t})$. From (3.1), we see that along $x=\xi\left(t ; x_{1}, \bar{t}\right), u(x, t)>0$. This infers that the backward characteristics $x=\xi\left(t ; x_{1}, \bar{t}\right)$ cannot $\operatorname{cross} x=a_{j}^{\epsilon}(t)$ and hence

$$
\xi\left(t ; x_{1}, \bar{t}\right)<a_{j}^{\epsilon}(t)
$$

Let $x_{1} \rightarrow a_{j}^{\epsilon}(\bar{t})-$. Due to the continuous dependence of solutions of ODEs on initial data,

$$
\xi\left(t ; x_{1}, \bar{t}\right) \rightarrow \xi\left(t ; a_{j}^{\epsilon}(\bar{t})-, \bar{t}\right)
$$

Then (3.5) leads to

$$
\xi\left(t ; a_{j}^{\epsilon}(\bar{t})-, \bar{t}\right) \leq a_{j}^{\epsilon}(t) .
$$


From (3.4), (3.7) and (3.1), we obtain the estimate

$$
\left|\frac{a_{j}^{\epsilon}(\bar{t})-a_{j}^{\epsilon}(t)}{\bar{t}-t}\right| \leq\left|\frac{a_{j}^{\epsilon}(\bar{t})-\xi\left(t ; a_{j}^{\epsilon}(\bar{t})-, \bar{t}\right)}{\bar{t}-t}\right| \leq \max _{u \in[0,1]} f^{\prime}(u)=f^{\prime}(1) .
$$

Corollary 3.2. If $x \notin \bigcup_{j=1}^{k}\left[a_{j}^{\epsilon}(t), b_{j}^{\epsilon}(t)\right]$, then $\xi^{\epsilon}(0 ; x, t) \notin \bigcup_{j=1}^{k}\left[a_{j}, b_{j}\right]$.

Lemma 3.3. For any sequence $\left\{\epsilon_{n}\right\}_{n=1}^{\infty}, \epsilon_{n} \rightarrow 0+$ as $n \rightarrow \infty$, there is a subsequence, also denoted by $\left\{\epsilon_{n}\right\}$ for simplicity, such that the limit

$$
u(\bar{x}, \bar{t})=\lim _{\epsilon_{n} \rightarrow 0+} u^{\epsilon_{n}}(\bar{x}, \bar{t})
$$

exists for almost all $(\bar{x}, \bar{t}) \in \mathbb{R} \times \mathbb{R}^{+}$. The range of $u(\bar{x} \pm, \bar{t})$ is $\{0,1\}$. Furthermore, there are uniform Lipschitzian curves $a_{j}(t)$ and $b_{j}(t) \equiv b_{j}$ defined $\left[0, T_{j}\right), j=$ $1,2, \ldots, n$, respectively such that $\left[a_{j}(t), b_{j}(t)\right] \subseteq\left[a_{j}, b_{j}\right], j=1,2, \ldots, n$, and that for each fixed $t>0$, the set

$$
Z(t):=\{x \in \mathbb{R} \mid u(x+, t)=0 \text { or } u(x-, t)=0\}=\bigcup_{j=1}^{n}\left[a_{j}(t), b_{j}(t)\right] .
$$

Proof. Since, by Lemma 3.1, the curves $a_{j}^{\epsilon_{n}}(t)$ and $b_{j}^{\epsilon_{n}}(t)$ defined on $\left[0, T_{j}^{\epsilon_{n}}\right]$ are Lipschitzian uniformly in $\epsilon_{n}>0$ and $j$, there is a subsequence of $\left\{\epsilon_{n}\right\}$, also denoted by $\left\{\epsilon_{n}\right\}$, such that

$$
a_{j}(t):=\lim _{n \rightarrow \infty} a_{j}^{\epsilon_{n}}(t), b_{j}(t):=\lim _{n \rightarrow \infty} b_{j}^{\epsilon_{n}}(t) \equiv b_{j},
$$

exits on $\left[0, T_{j}:=\lim _{n \rightarrow \infty} T_{j}^{\epsilon_{n}}\right]$ for all $j=1,2,3, \ldots, n$. By the definition of $a_{j}^{\epsilon_{n}}(t)$ and $b_{j}^{\epsilon_{n}}(t)$, we have

$$
\lim _{n \rightarrow \infty} u^{\epsilon_{n}}(x, t)=0
$$

for each $x \in \bigcup_{j=1}^{n}\left(a_{j}(t), b_{j}(t)\right)$. In the above statement and in the rest of this proof, if $t$ is out of the domain of the definition of $a_{j}(t)$ and $b_{j}(t)$, we just ignore $a_{j}(t)$ and $b_{j}(t)$.

It remains to prove that

$$
\lim _{n \rightarrow \infty} u^{\epsilon_{n}}(\bar{x}, \bar{t})=1
$$

for any

$$
\bar{x} \notin \bigcup_{j=1}^{k}\left[a_{j}(t), b_{j}(t)\right] .
$$

We observe that given $\bar{x}$ satisfying (3.13), if $n$ is large enough, then

$$
\bar{x} \notin \bigcup_{j=1}^{k}\left[a_{j}^{\epsilon_{n}}(\bar{t}), b_{j}^{\epsilon_{n}}(\bar{t})\right] .
$$

By Corollary 3.2,

$$
\xi^{\epsilon_{n}}(0 ; \bar{x}, \bar{t}) \notin \bigcup_{j=1}^{k}\left[a_{j}, b_{j}\right] .
$$


If $u^{\epsilon_{n}}(\bar{x}, \bar{t})=1$, there is no need of proving (3.12). If $u^{\epsilon_{n}}(\bar{x}, \bar{t}) \neq 1$, then we consider the minimal backward characteristic $\xi^{\epsilon_{n}}(t ; \bar{x}, \bar{t})$ of $(1.1)$ through the point $(\bar{x}, \bar{t})$. It satisfies

$$
\begin{aligned}
& \frac{d \xi}{d t}=f^{\prime}(v(t)) \\
& \frac{d v}{d t}=\frac{1}{\epsilon_{n}} v(1-v), \\
& (\xi(\bar{t}), v(\bar{t}))=\left(\bar{x}, u^{\epsilon_{n}}(\bar{x}-, \bar{t})\right) .
\end{aligned}
$$

According to Corollary 2.5, the solution of (3.16) is defined on $[0, \bar{t}]$. We integrate (3.16) to obtain

$$
u^{\epsilon_{n}}(\bar{x}-, \bar{t})=\frac{C_{\epsilon_{n}} \exp \left(\bar{t} / \epsilon_{n}\right)}{1+C_{\epsilon_{n}} \exp \left(\bar{t} / \epsilon_{n}\right)}
$$

where

$$
C_{\epsilon_{n}}:=\frac{u_{0}\left(\xi^{\epsilon_{n}}(0 ; \bar{x}, \bar{t})\right)}{1-u_{0}\left(\xi^{\epsilon_{n}}(0 ; \bar{x}, \bar{t})\right)}>0 .
$$

There are the following two possibilities for $C_{\epsilon_{n}}$ :

Case I.

$$
\liminf _{n \rightarrow \infty} C_{\epsilon_{n}}>0 \text {. }
$$

From (3.17), equation $u(\bar{x}, \bar{t})=\lim _{n \rightarrow \infty} u^{\epsilon_{n}}(\bar{x}-, \bar{t})=1$ holds in this case.

Case II. Condition (3.18) fails. In other words, there is a subsequence of $\left\{\epsilon_{n}\right\}_{n=1}^{\infty}$, still denoted by $\left\{\epsilon_{n}\right\}$, such that

$$
\lim _{n \rightarrow \infty} C_{\epsilon_{n}}=0+
$$

which is equivalent to

$$
\lim _{n \rightarrow \infty} u_{0}\left(\xi^{\epsilon_{n}}(0 ; \bar{x}, \bar{t})\right)=0
$$

in view of $(3.17 \mathrm{~b})$.

By the continuity of $u_{0}(x)$ and by further extracting a subsequence if necessary, we have

$$
\xi^{\epsilon_{n}}(0 ; \bar{x}, \bar{t}) \rightarrow a=a_{j} \text { or } b_{j} \text { as } n \rightarrow \infty
$$

for some $j \in\{1,2, \ldots, n\}$. Since $u^{\epsilon_{n}}(\bar{x}-, \bar{t})>0$, it is necessary in view of $(3.16)_{1}$ and $f^{\prime}(u)>0$ for $u>0$ that

$$
a \leq \bar{x}
$$

There are two cases according to (3.21) and (3.13):

Subcase II(1). $a<\bar{x}$. We calculate from (3.16) to obtain that

$$
\int_{u_{0}\left(\xi^{\epsilon_{n}}(0, \bar{x}, \bar{t})\right)}^{u^{\epsilon_{n}}(\bar{x}-, \bar{t})} \frac{f^{\prime}(u) d u}{u(1-u)}=\frac{\bar{x}-\xi^{\epsilon_{n}}(0, \bar{x}, \bar{t})}{\epsilon_{n}} \rightarrow \infty
$$

as $n \rightarrow \infty$. Note that $u=0$ is not a singular point for the integral in (3.23) since $f^{\prime}(0)=0$ and $f^{\prime}$ is differentiable. Then we have $u(\bar{x}, \bar{t})=\lim _{n \rightarrow \infty} u^{\epsilon_{n}}(\bar{x}-, \bar{t})=1$. 
Subcase $\mathrm{II}(2) . \bar{x}=a$.

For this case, we choose any $x_{1}<\bar{x}$ (or $x_{1}>\bar{x}$ ) close to $\bar{x}$. From (3.20), the closeness of $x_{1}$ to $a$ guarantees that $x_{1} \neq a_{j}$ or $b_{j}$ for any $j=1,2, \ldots, n$. Then our arguments for Subcase $\mathrm{II}(1)$ with $\bar{x}$ replaced by $x_{1}$ implies that $u\left(x_{1}, \bar{t}\right)=1$ and hence $u(\bar{x}-, t)=1$ (or $u(\bar{x}+, t)=1)$. Therefore $u(\bar{x}, \bar{t})=1$ in this case. 1.

Combining our analysis of Case I, Subcases $\mathrm{II}(1)$ and $\mathrm{II}(2)$, we conclude $u(\bar{x}, \bar{t})=$

Lemma 3.4. Let $u(x, t), a_{j}(t)$ and $b_{j}(t)$ be as in Lemma 3.3. Then the following hold:

(i) The curve $a_{j}(t)$ satisfies the Rankine-Hugoniot condition

$$
\frac{d a_{j}(t)}{d t}=\frac{f\left(u\left(a_{j}(t)-, t\right)\right)-f\left(u\left(a_{j}(t)+, t\right)\right)}{u\left(a_{j}(t)-, t\right)-u\left(a_{j}(t)+, t\right)}=f(1)-f(0) .
$$

$$
b_{j}(t)=b_{j}+f^{\prime}(0) t
$$

Proof. (ii) This is clear from Lemma 3.3.

(i) By the definition of $a_{j}(t)$, it is clear that

$$
u\left(a_{j}(t)-, t\right)=1, u\left(a_{j}(t)+, t\right)=0 .
$$

To prove $a_{j}(t)$ is determined by Rankine-Hugoniot condition for (1.1), it suffices to prove the jump discontinuity

$$
w(x, t):= \begin{cases}0, & \text { if } x>a_{j}(t) \\ 1, & \text { if } x<a_{j}(t)\end{cases}
$$

is a weak solution of

$$
u_{t}+f(u)_{x}=0 .
$$

To this end, we first prove that $a_{j}(\bar{t}) \geq a_{j}$. Indeed, if otherwise, there would be a $0<t<\bar{t}$ such that $a_{j}(t)<a_{j}$ sufficiently close to $a_{j}(0)=a_{j}$. There is a point $a_{j}(t)<x<a_{j}$ such that $u^{\epsilon_{n}}(x, t)=0$ for large $n$. This leads to $\xi^{\epsilon_{n}}(0 ; x, t)=x \in\left(b_{j-1}, a_{j}\right)$ and $u_{0}(x)=0$ which violates the definition of $a_{j}$ and $b_{j}$.

We further claim that $a_{j}(\bar{t})>a_{j}$. For this purpose, it suffices to prove $a_{j}(\bar{t}) \neq a_{j}$. We assume the contrary, $a_{j}(\bar{t})=a_{j}$ for some $\bar{t}>0$, for contradiction. For $x>a_{j}=$ $a_{j}(\bar{t})$ and close to $a_{j}, u^{\epsilon_{n}}(x, \bar{t})=0$ and hence $\xi^{\epsilon_{n}}(t ; x, \bar{t}) \equiv x$ and $u^{\epsilon_{n}}(x, t)=0$ for all $0<t<\bar{t}$ and hence $a_{j}(t)=a_{j}$ for $0<t<\bar{t}$. We pick a sufficiently small constant $\delta>0$. By definition of $a_{j}$ and $b_{j}$ in $\left(H_{2}\right)$, we know that $u_{0}(x) \neq 0$ in $\left(b_{j-1}, a_{j}\right)$. Then, the continuity of $u_{0}$ guarantees

$$
\eta:=\min _{x \in\left[\left(a_{j}+3 b_{j-1}\right) / 4, a_{j}-\delta\right]}\left(u_{0}(x)\right)>0 .
$$

For any $x_{1} \in\left[\left(a_{j}+b_{j-1}\right) / 2, a_{j}-\delta\right]$, the point $\xi^{\epsilon_{n}}\left(0 ; x_{1}, \bar{t} / 2\right)<x_{1}$. If $\xi^{\epsilon_{n}}\left(0 ; x_{1}, \bar{t} / 2\right) \geq$ $\left(a_{j}+3 b_{j-1}\right) / 4$, then (3.17) forces $u^{\epsilon_{n}}\left(x_{1}, \bar{t}\right) \rightarrow 1$ uniformly in $x_{1}$ as $\epsilon_{n} \rightarrow 0+$. If $\xi^{\epsilon_{n}}\left(0 ; x_{1}, \bar{t} / 2\right)<\left(a_{j}+3 b_{j-1}\right) / 4$, then, similar to $(3.23)$, we have

$$
\int_{u_{0}\left(\xi^{\epsilon}\left(0 ; x_{1}, \bar{t} / 2\right)\right)}^{u^{\epsilon_{n}}\left(x_{1}-, \bar{t} / 2\right)} \frac{f^{\prime}(u) d u}{u(1-u)}=\frac{x_{1}-\xi^{\epsilon_{n}}\left(0 ; x_{1}, \bar{t}_{1} / 2\right)}{\epsilon_{n}}>\frac{a_{j}-b_{j-1}}{4 \epsilon_{n}} \rightarrow \infty
$$


as $n \rightarrow \infty$. This again leads to $u^{\epsilon_{n}}\left(x_{1}, \bar{t}\right) \rightarrow 1$ uniformly in $x_{1}$ as $\epsilon_{n} \rightarrow 0+$. Thus, we proved that for $x_{1} \in\left[\left(a_{j}+b_{j-1}\right) / 2, a_{j}-\delta\right], u^{\epsilon_{n}}\left(x_{1}, \bar{t} / 2\right)$ is uniformly close to 1 for small $\epsilon_{n}>0$.

To prove the above claim, we consider the forward characteristics $\zeta^{\epsilon_{n}}(t):=$ $\zeta^{\epsilon_{n}}\left(t ; x_{1}, \bar{t} / 2\right)$ issued from

$$
\left(x_{1}=a_{j}-\delta, \bar{t} / 2\right) .
$$

Since the minimal backward characteristics issued from $\left(x=\xi^{\epsilon_{n}}\left(t ; \zeta^{\epsilon_{n}}\left(\bar{t}_{1}\right), \bar{t}_{1}\right), \bar{t}_{1}\right)$ with $\left(\bar{t}_{1}>\bar{t} / 2\right)$ cannot cross $x=\zeta^{\epsilon_{n}}(t)$ from the left as $t$ decreases (see Lemma $2.6)$, it holds that

$$
\xi^{\epsilon_{n}}\left(0 ; \zeta^{\epsilon_{n}}\left(\bar{t}_{1}\right), \bar{t}_{1}\right)<a_{j}-\delta .
$$

Then our arguments in the previous paragraph yield that $u^{\epsilon_{n}}\left(\zeta^{\epsilon_{n}}\left(\bar{t}_{1}\right), \bar{t}_{1}\right)$ is uniformly close to 1 for small $\epsilon_{n}>0$ and $\bar{t}_{1}>\bar{t} / 2$. The slope of $x=\zeta^{\epsilon_{n}}(t)$ is

$$
\frac{d \zeta^{\epsilon_{n}}}{d t}>f(1 / 2)-f(0)>0
$$

by virtue of Lemma 2.6 and $\left(H_{1}\right)$. Since $a_{j}^{\epsilon_{n}}(t) \rightarrow a_{j}(t)$ pointwisely as $n \rightarrow \infty$ and $a_{j}^{\epsilon_{n}}(t)$ is uniformly Lipschitzian, we have

$$
a_{j}^{\epsilon_{n}}(t) \rightarrow a_{j}(t) \equiv a_{j} \text { uniformly in } t \leq \bar{t} \text { as } n \rightarrow \infty .
$$

Then we can choose $\delta>0$ sufficiently small so that curves $x=\zeta^{\epsilon_{n}}(t)$ intersect $x=$ $a_{j}^{\epsilon_{n}}(t)$ at some $\bar{t}_{2}^{\epsilon_{n}} \in(\bar{t} / 2,3 \bar{t} / 4]$ when $\epsilon_{n}$ is small enough. We see that $a_{j}^{\epsilon_{n}}(t) \leq \zeta^{\epsilon_{n}}(t)$ for $t \in\left[\bar{t}_{2}^{\epsilon_{n}}, \bar{t}\right]$ because (3.33), (3.34) and $a_{j}^{\epsilon_{n}}(t)$ are uniformly Lipschitzian. As a consequence of this and (3.32), there is a constant $\delta_{1}>0$ such that $u^{\epsilon_{n}}(x, t)>1 / 2$ for $a_{j}^{\epsilon_{n}}(t)-\delta_{1}<x<a_{j}^{\epsilon_{n}}(t)$ and $\bar{t}_{2}^{\epsilon_{n}}<t<\bar{t}_{2}^{\epsilon_{n}}+\delta_{1}$. In other words, we have $u^{\epsilon_{n}}\left(a_{j}^{\epsilon_{n}}(t)-, t\right)>1 / 2$ and $u^{\epsilon_{n}}\left(a_{j}^{\epsilon_{n}}(t)+, t\right)=0$ for $\bar{t}_{2}^{\epsilon_{n}}<t<\bar{t}_{2}^{\epsilon_{n}}+\delta_{1}$. This leads to a contradiction: On one hand, the jump discontinuity at $x=a_{j}^{\epsilon_{n}}(t), \bar{t}_{2}^{\epsilon_{n}}<t<\bar{t}_{2}^{\epsilon_{n}}+\delta_{1}$, being a weak solution of (1.1), must propagate by a speed

$$
\frac{d a_{j}^{\epsilon_{n}}(t)}{d t}>(f(1 / 2)-f(0)) /(1 / 2)>0
$$

determined by Rankine-Hugoniot condition. On the other hand, the slope of $x=$ $a_{j}^{\epsilon_{n}}(t)$ is almost zero since $a_{j}^{\epsilon_{n}}(t) \rightarrow a_{j}$ as $n \rightarrow \infty$. This contradiction proves our claim that $a_{j}(t)>a_{j}$ for $t \in(0, \bar{t}]$.

To finish the proof of that (3.27) is a weak solution of (3.28), we consider the time interval $\left[t_{1}>0, t_{2}<T_{j}\right]$. From the definition $a_{j}^{\epsilon_{n}}(t)$, there is a constant $\delta_{2}>0$ such that

$$
u^{\epsilon_{n}}(x, t)=0 \quad \text { for } x \in\left(a_{j}^{\epsilon_{n}}(t), a_{j}^{\epsilon_{n}}(t)+\delta_{2}\right]
$$

for $t \in\left[t_{1}>0, t_{2}<T_{j}\right]$. Define

$$
\delta_{3}=\frac{1}{2} \min _{t \in\left[t_{1}, t_{2}\right]}\left(a_{j}^{\epsilon_{n}}(t)-a_{j}\right)>0 .
$$

For $\bar{t} \in\left[t_{1}, t_{2}\right]$ and $\bar{x} \in\left[a_{j}^{\epsilon_{n}}(\bar{t})-\delta_{3}, a_{j}^{\epsilon_{n}}(\bar{t})\right)$, it is necessary that

$$
\xi^{\epsilon_{n}}(0 ;, \bar{x}, \bar{t})<a_{j}
$$


for if otherwise, then $u^{\epsilon_{n}}(x, \bar{t})=0$ for all $x \in\left[\bar{x}, a_{j}^{\epsilon_{n}}(\bar{t})\right]$ which violates the definition of $a_{j}^{\epsilon_{n}}(\bar{t})$. Similar to $(3.23)$, we have

$$
\begin{aligned}
& M \int_{0}^{u^{\epsilon_{n}}(\bar{x}-, \bar{t})} \frac{d u}{1-u}>\int_{u_{0}\left(\xi^{\epsilon_{n}}(0, \bar{x}, \bar{t})\right)}^{u^{\epsilon_{n}}(\bar{x}-\bar{t})} \frac{f^{\prime}(u) d u}{u(1-u)} \\
& =\frac{\bar{x}-\xi^{\epsilon_{n}}(0, \bar{x}, \bar{t})}{\epsilon_{n}}>\delta_{3} / \epsilon_{n}
\end{aligned}
$$

where

$$
M:=\max _{u \in[0,1]} f^{\prime}(u) / u
$$

is finite since $f^{\prime}(0)=0$ and $f \in C^{2}$. Estimates (3.35) and (3.37) yield

$$
\frac{1}{\epsilon_{n}}\left|u^{\epsilon_{n}}(\bar{x}, \bar{t})\left(1-u^{\epsilon_{n}}(\bar{x}, \bar{t})\right)\right| \leq \frac{1}{\epsilon_{n}} \exp \left(-\frac{\delta_{3} \bar{t}}{M \epsilon_{n}}\right) \rightarrow 0
$$

as $n \rightarrow \infty$ uniformly for $\bar{t} \in\left[t_{1}, t_{2}\right]$ and $\bar{x} \in\left[a_{j}^{\epsilon_{n}}(t)-\delta_{3}, a_{j}^{\epsilon_{n}}(t)\right)$. Apply these estimates to the weak form of (1.1)

$$
\int_{0}^{T_{j}} \int_{\mathbb{R}}\left(-u \phi_{t}-f(u) \phi_{x}-\frac{1}{\epsilon_{n}} u^{\epsilon_{n}}\left(1-\left(u^{\epsilon_{n}}\right)^{2}\right) \phi\right) d x d t=0
$$

for test functions with compact support confined near $x=a_{j}(t), t_{1}<t<t_{2}$, one sees that the shock (3.27) is a weak solution of (3.28) for $t_{1}<t<t_{2}$. Due to the arbitrariness of $t_{1}, t_{2} \in\left(0, T_{j}\right),(3.27)$ is a weak solution of $(3.28)$ for $t \in\left(0, T_{j}\right)$. Thus, the Rankine-Hugoniot condition (3.24) holds for the shock (3.27).

Our above analysis already contains a complete picture of the structure of the limit of $u^{\epsilon_{n}}(x, t)$, the solution of (1.1). We see that the limit function $u(x, t)$ is completely determined by the curves $a_{j}(t)$ and $b_{j}(t), j=1,2, \ldots, n$. Furthermore, these curves are uniquely determined by (3.24) and (3.25), independent of the subsequence $\left\{\epsilon_{n}\right\}$. In other words, no matter how the subsequence $\left\{\epsilon_{n}\right\}$ is chosen, the limit functions $u(x, t)=\lim _{n \rightarrow \infty} u^{\epsilon_{n}}(x-, t)$ are the same. This proves the convergence of $u^{\epsilon}$ as $\epsilon \rightarrow 0+$. We summarize the above results in the following theorem:

Theorem 3.5. Let $u^{\epsilon}$ be the solution of (1.1). Under the assumption $\left(H_{1}\right)$ and $\left(H_{2}\right)$, the limit

$$
u(x, t)=\lim _{\epsilon \rightarrow 0+} u^{\epsilon}(x, t)
$$

exists for almost all $(x, t) \in \mathbb{R} \times \mathbb{R}^{+}$. The value of $u(x \pm, t)$ is either 1 or 0 . Furthermore, there are $n$ curves $a_{j}(t)$ and $b_{j}(t)$ defined on $\left[0, T_{j}\right], j=1,2, \ldots, n$, such that

(i) the set $\{x \in \mathbb{R} \mid u(x, t)=0\}=\bigcup_{j=1}^{n}\left(a_{j}(t), b_{j}(t)\right)$ and intervals $\left(a_{j}(t), b_{j}(t)\right)$, $j=1,2, \ldots, n$, are pairwise disjoint.

(ii) $b_{j}(t)=b_{j}$.

(iii) The curve $a_{j}(t)$ satisfies the Rankine-Hugoniot condition and hence

$$
a_{j}(t)=a_{j}+(f(1)-f(0)) t .
$$

(iv) At the end point $t=T_{j}$ of the domain of the definition of $a_{j}(t)$ and $b_{j}(t)$, $a_{j}\left(T_{j}\right)=b_{j}\left(T_{j}\right)$. 
Proof. Statements (i), (ii) and (iii) are already contained in Lemma 3.4. Statement (iv) is an immediate consequence of (ii) and (iii).

\section{Traveling waves of (1.1) With parabolic Regularization}

In this section we will study traveling waves of viscous regularization of (1.1):

$$
\partial_{t} u+\partial_{x} f(u)=\frac{1}{\epsilon} u(1-u)+A \epsilon \partial_{x x} u
$$

where $A>0$ is a constant. It is clear that (4.1) has two equilibria: $u=1$ and $u=0$. The traveling wave equation for (4.1) is

$$
\begin{aligned}
& -c u^{\prime}+f(u)^{\prime}=u^{\prime \prime}+u(1-u), \\
& u(-\infty)=1, u(+\infty)=0,
\end{aligned}
$$

where "'" denotes $\frac{d}{d y}$ with $y=(x-c t) / \epsilon$.

At the equilibrium point $u=1$, the eigenvalues of (4.2) are

$$
\begin{aligned}
& \lambda_{1,+}=\frac{1}{2 A}\left(f^{\prime}(1)-c+\sqrt{\left(f^{\prime}(1)-c\right)^{2}+4 A}\right)>0, \\
& \lambda_{1,-}=\frac{1}{2 A}\left(f^{\prime}(1)-c-\sqrt{\left(f^{\prime}(1)-c\right)^{2}+4 A}\right)<0,
\end{aligned}
$$

At $u=0$, the eigenvalues are

$$
\lambda_{0, \pm}=\frac{1}{2 A}\left(f^{\prime}(0)-c \pm \sqrt{\left(f^{\prime}(0)-c\right)^{2}-4 A}\right)<0 .
$$

It is clear that $\lambda_{0, \pm}<0$ if $c>f^{\prime}(0)+2 \sqrt{A}$.

Theorem 4.1. If $f^{\prime \prime}>0$ and

$$
c>f^{\prime}(1)+\sqrt{4 A+\left(f^{\prime}(1)-f^{\prime}(0)\right)^{2} / 4},
$$

then there is a traveling wave of (4.1) with speed $c>0$. Furthermore, if

$$
c<f^{\prime}(0)+2 \sqrt{A}
$$

then there is no traveling wave of (4.1) satisfying $0 \leq u \leq 1$.

Proof. If $c<f^{\prime}(0)$, then $\lambda_{0, \pm}>0$ and hence there is no traveling wave of (4.1). If $f^{\prime}(0) \leq c<f^{\prime}(0)+2 \sqrt{A}$, then the eigenvalue $\lambda_{0, \pm}>0$ is complex and hence the stable manifolds of (4.1) at $u=0$, if any, cannot stay inside the region $0 \leq u \leq 1$. The last statement then follows.

To prove the first statement of the theorem, we consider a triangle in the $\left(u, u^{\prime}\right)$ plane

$$
D:=\left\{\left(u, u^{\prime}\right) \in \mathbb{R}^{2} \mid 0 \leq u \leq 1, a u \leq u^{\prime} \leq 0\right\}
$$

where $a<0$ is a constant to be determined later. It is clear from (4.3) that there is an unstable manifold leaving $\left(u, u^{\prime}\right)=(1,0)$ and entering into $D$. We claim that with $a$ properly chosen, this unstable manifold cannot leave $D$ and hence goes to $(0,0)$. This claim shows that $(4.1)$ has a traveling wave. The boundary of $D$ consists of three pieces:

$$
\begin{aligned}
& \Gamma_{1}:=\left\{\left(u, u^{\prime}\right) \in \mathbb{R}^{2} \mid 0<u \leq 1, u^{\prime}=0\right\}, \\
& \Gamma_{2}:=\left\{\left(u, u^{\prime}\right) \in \mathbb{R}^{2} \mid u=1, a \leq u^{\prime}<0\right\},
\end{aligned}
$$




$$
\Gamma_{3}:=\left\{\left(u, u^{\prime}\right) \in \mathbb{R}^{2} \mid 0 \leq u \leq 1, u^{\prime}=a u\right\} .
$$

On $\Gamma_{1}, u^{\prime \prime}<0$ and hence the unstable manifold cannot cross $\Gamma_{1}$ from inside of $D$. To leave $D$ through $\Gamma_{2}$ requires $u^{\prime}>0$, which is not possible on $D$. Now it remains to prove that the unstable manifold cannot leave $D$ through $\Gamma_{3}$. To this end, we compute from (4.2) to get

$$
\frac{d u^{\prime}}{d u}=\frac{\left(f^{\prime}(u)-c\right) u^{\prime}-u(1-u)}{A u^{\prime}} .
$$

On $\Gamma_{3}$, we have

$$
\begin{aligned}
\frac{d u^{\prime}}{d u}-a= & \frac{1}{A a}\left[-A a^{2}+\left(f^{\prime}(u)-c\right) a-(1-u)\right] \\
= & -\frac{1}{a}\left[a-\frac{1}{2}\left(\frac{f^{\prime}(u)-c}{A}+\sqrt{\frac{\left(f^{\prime}(u)-c\right)^{2}}{A^{2}}-\frac{4(1-u)}{A}}\right)\right] \\
& \times\left[a-\frac{1}{2}\left(\frac{f^{\prime}(u)-c}{A}-\sqrt{\left.\frac{\left(f^{\prime}(u)-c\right)^{2}}{A^{2}}-\frac{4(1-u)}{A}\right)}\right] .\right.
\end{aligned}
$$

Recalling the assumption $\left(H_{1}\right), f^{\prime \prime}>0$, we have the following estimates for $u \in$ $[0,1]$ :

$$
\begin{aligned}
& \frac{f^{\prime}(u)-c}{A}+\sqrt{\frac{\left(f^{\prime}(u)-c\right)^{2}}{A^{2}}-\frac{4(1-u)}{A}} \\
& >\frac{f^{\prime}(0)-c}{A}+\sqrt{\frac{\left(f^{\prime}(1)-c\right)^{2}}{A^{2}}-\frac{4}{A}}=: a_{+},
\end{aligned}
$$

and

$$
\begin{aligned}
& \frac{f^{\prime}(u)-c}{A}-\sqrt{\frac{\left(f^{\prime}(u)-c\right)^{2}}{A^{2}}-\frac{4(1-u)}{A}} \\
& <\frac{f^{\prime}(1)-c}{A}-\sqrt{\frac{\left(f^{\prime}(1)-c\right)^{2}}{A^{2}}-\frac{4}{A}}=: a_{-}
\end{aligned}
$$

Under the condition (4.5), we can prove that

$$
0>a_{+}>a_{-} .
$$

Choose $a$ between $a_{-}$and $a_{+}$. Then (4.11) yields that at $\Gamma_{3}$, where $u^{\prime}=a u$, the difference of slope of the unstable trajectory and that of $\Gamma_{3}$

$$
\frac{d u^{\prime}}{d u}-a<0
$$

This proves our claim.

Corollary 4.2. Let

$$
c>f^{\prime}(1)+\sqrt{4 A+\left(f^{\prime}(1)-f^{\prime}(0)\right)^{2} / 4}
$$

hold and let $u(y)$ be traveling waves provided by Theorem 4.1. Then $u(y)$ satisfies

$$
u(y)=O(1) \exp \left(\lambda_{0,+} y\right) \text { as } y \rightarrow \infty,
$$

where $\lambda_{0,+}$ is given in (4.4). 
Proof. Let $u(y)$ be the traveling wave of (4.1) given by the last theorem. A computation on (4.2) shows that near $u=0$,

$$
\frac{d u^{\prime}}{d u}=\lambda_{0, \pm}
$$

On the other hand, from the proof of the last theorem, we know that

$$
\frac{d u^{\prime}}{d u}>a>a_{-}>\lambda_{0,-} .
$$

Thus, only $\frac{d u^{\prime}}{d u}=\lambda_{0,+}$. is possible. In other words, the stable trajectory $u(y)$ corresponds to the eigenvalue $\lambda_{0,+}$ and hence the conclusion holds.

\section{REFERENCES}

[AK] S. Allen and J. Cahn, A microscopic theory for antiphase boundary motion and its application to antiphase domain coarsening, Acta Metallurgica 27, 1084-1095, 1979.

[BKT] A.C. Berkenbosch, E.F. Kaasschieter, and J.H.M. ten Thije Boonkkamp, The numerical wave speed for one-dimensional scalar hyperbolic conservation laws with source terms, RANA 94-01, Eindhover Univ. of Tech., 1994.

[BJ] W.Z. Bao and S. Jin, The random projection method for hyperbolic systems with stiff reaction terms, J. Comp. Phys. 163, 2000, 216-248. CMP 2000:17

[Br] M. Bramson, Convergence of solutions of Kolmogorov equation to traveling waves, Memoirs AMS, vol. 44, No 285 (1983). MR 84m:60098

[CMR] P. Colella, A. Majda and V. Roytburd, Theoretical and numerical structure for reacting shock waves, SIAM J. Sci. Stat. Comp. 7, 1986, 1059-1080. MR 87i:76037

[Daf] C. M. Dafermos, Generalized characteristics and structure of solutions of hyperbolic conservation laws, Indiana Univ. Math. J. 26, 1097-1119, 1977. MR 56:16151

[Fan] H. Fan, Traveling waves, Riemann problems and computations of a model of the dynamics of liquid/vapor phase transitions, J. Diff. Eqs., 150 (1998) 385-437. MR 99j:35131

[FH1] H. Fan and J. K. Hale, Large time behavior in inhomogeneous conservation laws, Arch. Rational Mech. Anal., 125, 201-216, 1993. MR 94k:35187

[FH2] H. Fan and J. K. Hale, Attractors in inhomogeneous conservation laws and parabolic regularizations, Trans. Ameri. Math. Soc. 347, 1239-1254, 1995. MR 95g:35114

[Fil] A. F. Filippov, Differential equations with discontinuous right-hand side, Mat. Sb. (N.S.)42, 99-128; English Transl., Amer. Math. Soc. Transl. Ser. 2, Amer. Math. Soc., Providence, RI, 1960.

[FJT] H. Fan, S. Jin and Z-H Teng, Zero reaction limit for hyperbolic conservation laws with source terms, to appear in J. Diff. Eqs.

[Har] J. Härterich, Heteroclinic orbits between rotating waves in hyperbolic balance laws, Proc. Roy. Soc. Edinburgh Sect. A, 129 (1999), 519-538. MR 2000e:35136

[Kru] S.N. Kruzhkov, First order quasilinear equations in several independent variables, Math. USSR-Sb. 10, 217-243, 1970.

[Lyb] A. N. Lyberopoulos, A Poincarè-Bendixson theorem for scalar conservation laws, Proc. Roy. Soc. Edinburgh, 124A, 589-607, 1994.

[LY] R.J. LeVeque and H.C. Yee, A study of numerical methods for hyperbolic conservation laws with stiff source terms, J. Comp. Phys. 86, 1990, 187-210. MR 90k:76009

[Mas] C. Mascia, Traveling wave solutions for a balance law, Proc. Roy. Soc. Edinburgh Sect. A 127, 567-593, 1997. MR 98c:35105

[MS] C. Mascia and C. Sinestrari, The perturbed Riemann problem for a balance law, Adv. Diff. Eqs. 9, 779-810, 1997. MR 2001e:35115]

[Sin1] C. Sinestrari, Large time behavior of solutions of balance laws with periodic initial data, Nonl. Diff. Appl. 2, 111-131, 1995. MR 96a:35112

[Sin2] C. Sinestrari, Asymptotic profile of solutions of conservation laws with source, Differential Integral Equations 9, 499-525, 1996. MR 96m:35206 
[Sin3] C. Sinestrari, Instability of discontinuous traveling waves for hyperbolic balance law, J. Diff. Eqn. 134, 269-285, 1997. MR 97m:35170

[VK] W.G. Vincenti and C.H. Kruger, Jr., Introduction to Physical Gas Dynamics, Krieger, 1975.

Department of Mathematics, Georgetown University, Washington, D.C. 20057

E-mail address: fan@math.georgetown.edu 